\title{
Retraction: Application of Amphibious Technology in the ReutoMail
}

\author{
Sun Ping \\ Zhejiang Water Conservancy and Hydropower College \\ Hangzhou, P.R. China 310018 \\ htbbs3a163.com
}

Several conference proceedings have been infiltrated by fake submissions generated by the SCIgen computer program. Due to the fictional content the chapter "Application of Amphibious Technology in the ReutoMail" by "Sun Ping" has been retracted by the publisher. Measures are being taken to avoid similar breaches in the future. 